\title{
Negative priming is not task bound: A consistent pattern across naming and categorization tasks
}

\author{
DAN L. CHIAPPE and COLIN M. MACLEOD \\ University of Toronto, Scarborough Campus, Scarborough, Ontario, Canada
}

\begin{abstract}
When a word that was a to-be-ignored flanker on an initial prime trial becomes the target on the subsequent probe trial, responding to that word on the probe trial is slowed, a phenomenon called negative priming. Virtually all prior studies have required subjects to perform the same task on both the prime and the probe trials. Thus, the extent to which negative priming is task bound is uncertain. We manipulated task factorially on the prime and probe trials, resulting in four groups: name-name, name-categorize, categorize-name, and categorize-categorize. The results showed equivalent negative priming of about $22 \mathrm{msec}$ both within and between tasks for identical words, but no negative priming for semantically related words from the same category. These findings suggest (1) that negative priming for identical words can readily cross task types; and (2) that semantic negative priming does not occur for words, at least when categorical relatedness alone determines the semantic relation.
\end{abstract}

Among researchers studying selective attention, it is now widely held that, to select successfully, attention requires the active inhibition of task-irrelevant information (Driver \& Tipper, 1989; Neill, 1977; Tipper, 1985; Tipper \& Cranston, 1985; Tipper \& Driver, 1988). To select for one stimulus or stimulus dimension, we must simultaneously select against others. Ignoring an item on one trial (the prime trial) makes it more difficult to process that item on the very next trial (the probe trial). This interference can last up to several seconds, as studies by Neill and Valdes (1992) and Tipper, Weaver, Cameron, Brehaut, and Bastedo (1991) reveal. This inhibitory effect, first observed by Dalrymple-Alford and Budayr (1966), has been termed the distractor suppression effect (Neill, 1977) or, more widely, negative priming (Tipper, 1985). Fox (1995) and May, Kane, and Hasher (in press) have recently reviewed the now-burgeoning negative-priming literature.

Negative priming is now so well documented that research has shifted from demonstrating such inhibitory effects to discovering their exact locus. Specifically, does the inhibition apply to a particular response, or does it operate upon a central representation of the stimulus? Tipper and his colleagues suggest that it is a more cen-

\footnotetext{
This research was supported by Grant A7459 from the Natural Sciences and Engineering Research Council of Canada. We thank Douglas Bors, Penny Chiappe, Bert Forrin, Michael Masson, and Mark Schmuckler for their helpful advice, and James Neely, Trammell Neill, Steven Tipper, and Elaine Fox for their thoughtful reviews. Correspondence concerning this article may be addressed to $\mathrm{D}$. L. Chiappe or C. M. MacLeod, Division of Life Sciences, University of Toronto, Scarborough Campus, Scarborough, ON, Canada M1C 1A4 (e-mail: danilo@psych.utoronto.ca; macleod@lake.scar.utoronto.ca).
}

tral representation that is inhibited by selective attention. As one illustration, Tipper (1985) observed negative priming using superimposed pictures of objects. Subjects took longer to name a picture on the probe trial if it had appeared as the to-be-ignored picture on the immediately preceding prime trial than they did if it had not. Moreover, this pattern recurred for semantically related pictures, indicating a spread of inhibition to members of the same semantic category. Selective attention seems to involve inhibition of the representation of the particular ignored stimulus and also - at least in the case of pictures - of semantically related stimuli.

Using the other prevalent task, that of categorization, Tipper and Driver (1988) took this a step farther, observing negative priming for conceptually identical items across stimulus domains. They found that, whether the stimulus was a word or picture, ignoring an item on the prime trial caused interference with processing that same item on the probe trial. So, for example, ignoring a picture of a foot on one trial interfered with categorizing the word foot on the next trial. Moreover, for pictures, semantic negative priming was observed between members of a category, replicating the findings of Tipper (1985). Intriguingly, however, there was no semantic negative priming for categorically related words.

Tipper, MacQueen, and Brehaut (1988) reported further evidence consistent with the claim that the locus of inhibition is not a particular peripheral response, but, rather, is a central representation of the irrelevant information. Their subjects had to indicate the identity of a target letter presented with an irrelevant distractor letter. The subjects responded in one of four ways: (1) by a keypress on both the prime and the probe trials; (2) by an 
oral response on both the prime and the probe trials; (3) by an oral response on the prime trial and a keypress on the probe trial; or (4) by a keypress on the prime trial and an oral response on the probe trial. They found that ignoring a letter on the prime trial caused interference in processing that letter if it then became the target in the probe display. Moreover, negative priming did not depend on the modality of the response required to the prime and probe displays (i.e., on whether it was an oral response or a key response). This evidence strengthened the argument that negative priming does not take place at the level of a particular peripheral response.

Neill, Lissner, and Beck (1990) extended this claim using a matching task to uncouple stimulus and response effects. Their subjects had to say whether the second and fourth letters in an array of five letters were the same or different. The other three positions all contained the same irrelevant letter. The irrelevant letters on the prime trial were either the same as or different from one or both of the target letters on the probe trial. Hence, if a central representation is inhibited, "negative priming should not depend on whether the current response is the same as that made in the previous trial" (Neill et al., 1990, p. 398). In fact, although negative priming occurred when the ignored letters on the prime trial became target letters on the probe trial, the response match between the prime and probe trials did not matter. Response-modality match had no impact, consistent with the findings of Tipper et al. (1988).

These studies support the conclusion that negative priming derives from the active inhibition of centralized representations of task-irrelevant information. If the locus of inhibition is central, however, it should not be task bound: Negative priming should occur across different tasks, provided that the probe task uses the same representation as the one that was inhibited during the prime task, or perhaps a semantically related one. Unfortunately, in virtually all of the published studies, the subjects did the same task on both the prime and probe trials, rendering uncertain the extent to which negative priming is task bound.

There are two closely related exceptions. Yee (1991, Experiment 1) studied the fate of ignored information using different prime and probe tasks. The subjects classified geometric stimuli according to disjunctive rules on the prime trial and then made lexical decisions about a target word on the probe trial. In addition to the geometric stimulus, the prime display included either one or two irrelevant words. The target display consisted of either a strong associate of one of the ignored primes, an unrelated word, or a nonword. Yee found no negative priming when the prime display contained only a single word. However, she found semantic negative priming in the double-word condition: The subjects took longer to make lexical decisions to the single target word when it was associatively related to one of the two ignored prime words than they did when it was unrelated to both. Fox (1994, Experiment 3) replicated this finding when sub- jects categorized a target number on the prime trial while ignoring distracting words, and then made a lexical decision on the probe trial.

The discovery by Yee (1991) and Fox (1994) of semantic negative priming with words is interesting because of the inconsistency with which this has been reported in the literature. As mentioned, Tipper and Driver (1988) failed to find significant semantic negative priming for words. Moreover, Fuentes and Tudela (1992, Experiment 1) found positive semantic priming from previously unattended prime words in a lexical-decision task, especially when the retinal eccentricity of the distractors was fairly high $\left(4.3^{\circ}\right)$. In their second experiment, they found semantic negative priming only when the unattended word was displaced $2^{\circ}$ from the attended word in the prime display. There was a trend toward semantic negative priming when the unattended prime word was displaced $3.6^{\circ}$. Other studies have failed to find any priming effectseither positive or negative-from unattended primes related to the target (Dark, Johnston, Myles-Worsley, \& Farah, 1985; Inhoff, 1982; Inhoff \& Rayner, 1980). In short, the status of semantic negative priming for words remains unclear.

With these studies as context, and with the particular impetus of Yee's (1991) experiment, we explored the extent to which negative priming depends on the task that the subject performs during the prime and the probe trials. As in much of the literature, we used only words as stimuli on all trials. We also used the two most prevalent tasks, naming and categorization, which we manipulated factorially on the prime and probe trials. This resulted in four between-subject groups: name-name, categorizename, name-categorize, and categorize-categorize. We also manipulated the relation between the ignored word on the prime trial and the target word on the probe trial. This yielded three within-subject conditions: (1) identi$c a l$, in which the ignored prime word is identical to the target probe word; (2) related, in which the ignored prime is from the same semantic category as the target probe word; and (3) unrelated, in which the ignored prime is from a different semantic category from that of the target probe word.

Our design differed from that of Yee (1991) in at least four key respects. First, both naming and categorization appeared as prime and probe tasks, depending upon the condition, whereas Yee used only the geometric classification task as her prime task and only the lexical decision task as her probe task. Our design permits us to determine the degree to which negative priming is reciprocal across tasks. Second, unlike Yee's study, ours was designed to assess both semantic negative priming and identity negative priming across tasks, permitting a direct comparison between them. If negative priming does span tasks, does this apply differently to the word itself than to related words? A clear answer to this question does not seem to be available from the literature. Third, Yee used different target stimuli on the prime and probe trials-geometric stimuli for primes, and words and non- 
words for probes. We used only words on both trials, thereby holding the stimuli constant and varying only the task. Finally, Yee's subjects responded with a binary keypress response on each trial, whereas our subjects responded vocally. Vocal responding to a number of different stimuli eliminates potential compatibility effects across successive keypresses, in addition to generalizing to another response modality.

\section{METHOD}

\section{Subjects}

Eighty-four undergraduates were recruited from the Scarborough campus of the University of Toronto. For taking part, they were given bonus points in their introductory psychology course. Using as a criterion the preset maximum error rate of .20 , we excluded 5 subjects Because of unequal sample sizes in the four between-subject combinations, 7 additional subjects were randomly excluded, to leave 18 subjects in each of the four combinations-a total of 72 subjects.

\section{Apparatus}

Testing was carried out on IBM-compatible 286 computers equipped with Tatung CM-1496 14-in. VGA color monitors. When a subject spoke into a Realistic Highball-7 microphone, the signal was amplified by a Realistic SA-150 stereo amplifier, and input through a specially modified keyboard as if the hyphen key had been pressed. All programming was done in QuickBASIC 4.5 with millisecond-accuracy timing routines taken from Graves and Bradley $(1987,1988)$. The screen background color was black (Palate \#0), and the instructions were presented in white (Palate \#15). The words to be attended were presented in blue (Palate \#9); the words to be ignored were presented in white. The patternmasking stimuli and the fixation points were also presented in white. All materials were printed in regular lowercase font with 80 characters per line. The subjects sat about $40 \mathrm{~cm}$ from the screen.

\section{Stimuli}

The stimuli consisted of 10 words, 2 from each of five categories, selected from the Battig and Montague (1969) norms. The words (with their category and rank in that category in parentheses) were: (musical instrument [shortened to music]) banjo (14), fiddle (27); (furniture) couch (7), dresser (8); (tool) hatchet (34), pliers (10); (body part [shortened to body]) heart (15), stomach (12); and (animal) giraffe (14), mouse (10). To minimize possible floor effects in priming that may result from using highly ranked words, none of ours ranked higher than seventh in the norms. The same 10 words were used throughout the experiment, following much of the tradition (e.g., Tipper, 1985; Tipper \& Driver, 1988). It should be noted, however, that negative priming can occur without repeating the same small set of words throughout the experiment (Yee, 1991; but see Malley \& Strayer, 1995).

\section{Procedure}

The experiment consisted of 10 pairs of practice trials and 90 pairs of experimental trials in blocks of 10 . The subjects could rest after each block and resume by asking the experimenter to proceed. Each pair of trials consisted of a prime trial followed by a probe trial. Prior to the prime trial, the subjects were cued with a 600 -msec fixation of " ++ " on each of Lines 12 and 13 at the center of the screen. The prime display followed immediately and consisted of two words, located where the two fixation stimuli had been. The subject was instructed to attend to the blue word and to ignore the white word; cuing by color is in keeping with much of the literature (e.g., Tipper, 1985; Tipper \& Cranston, 1985). The location of the cued word was randomized and was equally likely to be in either the top or the bottom position over trials, preventing subjects from guessing where the target word would appear.

The subjects were randomly assigned to one of the four betweensubject conditions defined by the task they had to perform on the cued word in the prime and probe displays. In the name-name condition, the subjects had to read aloud the cued word in both the prime and the probe displays. In the categorize-categorize condition, the subjects categorized aloud both the cued prime and the cued probe, using the specified category names. In the name-categorize condition, the subjects had to read aloud the cued prime word and then categorize aloud the cued probe word. In the categorize-name condition, the subjects categorized aloud the cued prime and then read aloud the cued probe. The first two conditions should replicate established negative priming within single tasks. The latter two conditions investigate negative priming across tasks.

The subjects were instructed to respond as quickly as possible to the cued prime word by either reading it or categorizing it aloud. The prime words remained on the screen until the subject responded. Immediately upon response, a 100-msec masking display appeared that consisted of two rows of seven $X$ s (i.e., $X X X X X X X X$ ), one row above the other. They appeared in the same location in which the signaled and unsignaled words of the prime display had appeared. The masking stimulus was used to minimize any contribution of the iconic image produced by the irrelevant word, thereby discouraging subjects from switching their attention to the irrelevant word once a response had been made.

The pattern mask was followed by another fixation point presented for $500 \mathrm{msec}$. Thus, the delay between the prime display and the target display was $600 \mathrm{msec}$, a delay that should produce a high degree of inhibition, according to studies by Neill and Valdes (1992) and Tipper et al. (1991). This delay was followed by the probe display, which also consisted of two words presented one above the other, in the same location as the prime and the mask. Again, the target word appeared in blue and the to-be-ignored word appeared in white.

The probe display appeared until the subject responded, depending on the condition, by either reading or categorizing the cued word aloud into the microphone. The time to perform the probe task was measured by the latency between the onset of the probe display and the subject's vocal response. The probe display was followed by a masking stimulus, which lasted $600 \mathrm{msec}$. After the masking stimulus, there was a blank interval of $1,500 \mathrm{msec}$, which ended with the question "Ready?" appearing at the center of the screen on Line 12. This served to warn the subject about the onset of the next pair of trials, as well as to allow the experimenter to record the accuracy of the subject's responses on the preceding pair of trials. The question "Ready?" remained on the screen until the experimenter entered the response accuracy, at which point the next trial began.

Trials in each of the four conditions were of three types, reflecting the different relations between the to-be-ignored word in the prime display and the target word in the probe display. In the unrelated conditionthe control condition - the ignored word was neither identical to the cued word in the probe display, nor was it semantically related to it (i.e., in the same category). For example, the subject might have had to ignore hatchet in the prime display and then to either read or categorize dresser in the probe display. In the identical condition, the ignored word in the prime display was identical to the target word in the probe display. For example, if the subject had to ignore hatchet in the prime display, he or she would have to either read or categorize hatchet in the probe display. Finally, in the related condition, the cued word in the probe display was from the same semantic category as the ignored word in the prime display. For example, if the subject ignored pliers in the prime display, he or she would have to either read or categorize hatchet in the probe display. The subjects saw 30 items from each of these three within-subject conditions, with the three conditions randomized over trials. Within a display, the ignored word and the cued word were always from different categories; further, the ignored word in the probe display was always from a different category than either of the words in the prime display. Using the identical condition as an illustration, in the prime display, fiddle could be cued and dresser ignored; then, in the probe display, dresser would be cued and hatchet could be ignored.

\section{RESULTS}

Table 1 presents the mean response latencies and the mean proportions of errors for all conditions. The latencies are means of individual-subject untrimmed means; the errors represent all trials on which either or both of 
Table 1

Mean Response Latencies (in Milliseconds) and Mean

Proportions of Errors (PE) as a Function of the Prime Task, the Probe Task, and the Relation Between the Ignored Prime Word and the Target Probe Word

\begin{tabular}{|c|c|c|c|c|c|c|}
\hline \multirow[b]{3}{*}{ Prime-Probe Task } & \multicolumn{6}{|c|}{ Word Relation } \\
\hline & \multicolumn{2}{|c|}{ Identical } & \multicolumn{2}{|c|}{ Related } & \multicolumn{2}{|c|}{ Unrelated } \\
\hline & $M$ & PE & $M$ & PE & $M$ & PE \\
\hline Name-name & 670 & .022 & 653 & .028 & 653 & .024 \\
\hline Categorize-name & 712 & .070 & 684 & .076 & 691 & .080 \\
\hline Name-categorize & 993 & .054 & 961 & .048 & 967 & .057 \\
\hline Categorize-categorize & 1,026 & .070 & 998 & .044 & 1,002 & .078 \\
\hline
\end{tabular}

the prime and probe responses were incorrect. For easier inspection, and because negative priming was the focus of this research, negative priming difference scores (identical - unrelated; related - unrelated) are presented in Figure 1.

Two analyses of variance (ANOVAs) were performed on the latency data. The first was a $3 \times 2 \times 2$ mixed analysis that examined word relation (identical, related, and unrelated) as a function of prime task (name vs. categorize) and probe task (name vs. categorize). The second ANOVA was a $2 \times 2 \times 2$ ANOVA that also involved the two primeprobe-task variables, but that now used the negative priming difference scores, permitting us to compare directly the amount of negative priming in the identical condition with that in the related condition. For completeness, the corresponding $2 \times 2 \times 2$ ANOVA is also reported for the error data.

\section{Latency Analyses}

The main findings concern the response latencies. These analyses yielded straightforward results. In the $3 \times$ $2 \times 2$ analysis, the main effect of word relation was significant $\left[F(2,136)=9.95, M S_{\mathrm{e}}=1417.62, p<.001\right]$, with identical words $(850 \mathrm{msec})$ slower than either related $(824 \mathrm{msec})$ or unrelated $(828 \mathrm{msec})$ words. Not surprisingly, there was also a significant effect of probe type $\left[F(1,68)=141.62, M S_{\mathrm{e}}=37628.74, p<.001\right]$, with categorization $(991 \mathrm{msec}$ ) much slower than naming $(677 \mathrm{msec})$. The small advantage for naming $(816 \mathrm{msec})$ over categorization $(854 \mathrm{msec})$ as a function of prime type was not reliable $[F(1,68)=1.83, p=.18]$. None of the interactions approached significance (all $F \mathrm{~s}<1$ ).

The principal analysis - the $2 \times 2 \times 2$ ANOVA directly examining the negative priming difference scoresshowed that the negative priming in the identical condition $(22 \mathrm{msec})$ was reliably greater than that in the related condition (which actually showed $4 \mathrm{msec}$ of positive priming) $\left[F(1,68)=14.11, M S_{\mathrm{e}}=1739.14, p<\right.$ $.001]$. There were no main effects of prime- or probe-task type $(F \mathrm{~s}<1)$, nor did any of the interactions approach significance (all $F_{\mathrm{S}}<1$ ).

Across the four prime-probe-task groups ( $n=18$ in each), identity negative priming was shown by the majority of subjects ( 15 of the name-name subjects, 13 of the categorize-name subjects, 12 of the name-categorize subjects, and 15 of the categorize-categorize subjects). In contrast, as would be expected by chance, semantic negative priming appeared only in about half of the subjects in each prime-probe group (10 of the name-name subjects, 8 of the categorize-name subjects, 8 of the namecategorize subjects, and 8 of the categorize-categorize subjects).

Put simply, we observed reliable and consistent negative priming for identical words of about $22 \mathrm{msec}$. This was unaffected by the task performed on either the first or the second trial of a pair. In contrast, the $4 \mathrm{msec}$ of positive priming for related words was not reliably different from zero, and was reliably below the negative priming for identical words, as Figure 1 clearly shows.

\section{Error Analyses}

The error data are of little interest, so we present the $2 \times$ $2 \times 2$ ANOVA only to be complete. This analysis shows that there was no negative priming evident for either the identical or the related words in the error data. Thus, effects in the latency data could not be the result of speedaccuracy tradeoffs. There was no suggestion of negative priming, either in the identical errors $(-.006)$ or in the related errors $(-.011 ; F<1)$, nor-except for the prime type $\times$ identical-related interaction $[F(1,68)=2.68$, $\left.M S_{\mathrm{e}}=.002, p=.11\right]$ - were any other effects even close to significant (all $F \mathrm{~s}<1$ ).

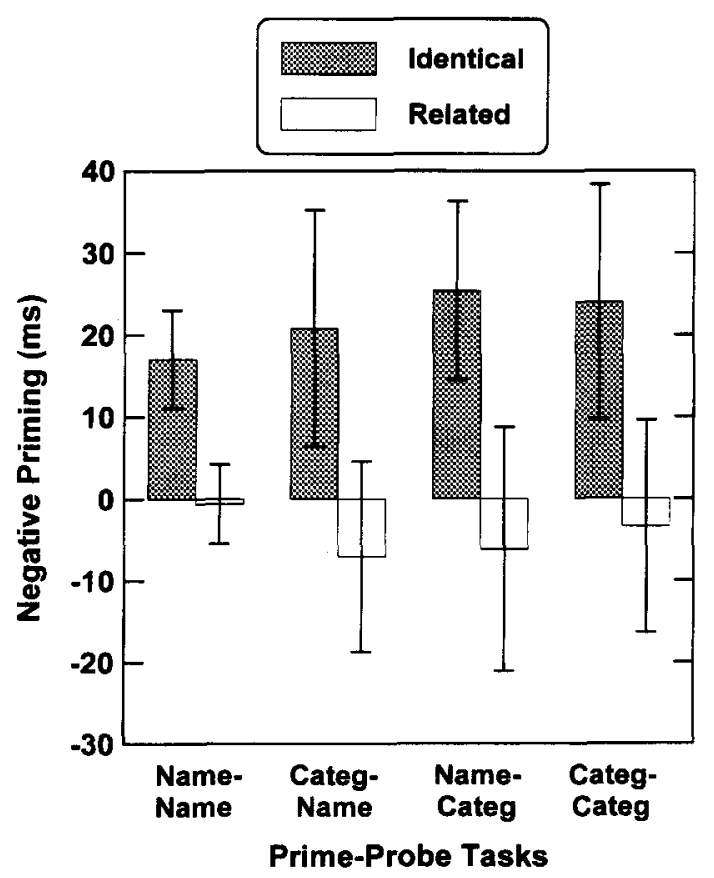

Figure 1. The amount of negative priming in each of the four prime-probe-task combinations, shown separately for the identical and related conditions. These difference scores were obtained by subtracting the unrelated condition mean on the probe trial from the identical or related condition mean on the prime trial. Error bars are standard errors of their respective means. Only the negative priming in each of the four identical word conditions is statistically reliable; there was no negative priming for any of the related word conditions. 


\section{DISCUSSION}

Negative priming occurs across stimulus modalities (e.g., picture and word; Tipper \& Driver, 1988) and response modalities (e.g., keypress and vocal; Tipper et al., 1988). It not only occurs for identical items (e.g., letters; Tipper \& Cranston, 1985), it can also occur for related items (e.g., pictures from the same semantic category; Tipper, 1985). In addition, it is independent of the match between the response choices on the prime and probe trials (e.g., as in the same-different matching task; Neill et al., 1990). Most recently, Yee (1991) and Fox (1994) have even suggested that negative priming may span different prime and probe tasks, at least when there were two to-be-ignored words on the prime trial to which the target word on the probe trial could be related.

Our study demonstrates that negative priming readily occurs across tasks virtually unscathed, but only for identical words. For semantically related words that are members of the same category, we failed to find negative priming, either within the same task or between tasks. These results are noteworthy for several reasons. First, we used the two most studied tasks - naming and categorization. Second, we factorially combined them, and showed that task order was unimportant. Third, within the same experiment, we demonstrated a dissociation between two types of materials: Only identical words-not categorically related words - caused negative priming in our experiment, and they did so consistently. Fourth, our results undermine the assumption that response repetition is the key to negative priming: The presence of identity priming across tasks shows that such repetition is not necessary; the absence of semantic priming within task shows that such repetition is not sufficient.

The results of the present study can be explained by any one of three competing general theories of negative priming. The first is Tipper's (1992; Tipper, Weaver, \& Houghton, in press) behavioral goals theory of inhibition. Tipper argues that the mechanisms of inhibition are flexible, adjusting to meet different task demands. Only those properties of the distractor that directly compete with the target in terms of the goals to be achieved will be inhibited. In the context of our study, when the subjects had to name the target word and ignore the distractor in the prime display (the name-name and name-categorize conditions), they would have inhibited the lexical representation of the distractor because it could interfere with the naming of the target. Further, in the categorize-name and categorize-categorize conditions, the subjects may also have inhibited only the lexical representation of the prime distractor because this may have been sufficient to allow them to carry out the categorization task. Thus, naming or categorizing the cued prime word would be accomplished through the selective inhibition of the lexical representation of the distractor. This would yield the overall clear pattern of identity negative priming without semantic negative priming that we found.

Another possible explanation of the results is the feature mismatch theory (Milliken, Tipper, \& Weaver, 1994; Park \& Kanwisher, 1994; Tipper et al., in press). According to this theory, negative priming does not result from the inhibition of distracting information, contrary to the traditional assumption; instead, it results from a mismatch in the features of the representations of the target item in the probe display and the distractor item in the prime display. That is, the subjects construct representations of objects in the visual array, including the ignored prime items. These representations include information about features, such as color and size of the object. In processing the probe display, the subject attempts to match the target to recently experienced items. If there is a complete match, processing of the target is facilitated. If there is only a partial match (e.g., if one of the properties of the item is different), processing is impeded by the presence of the mismatching information, yielding negative priming.

The feature mismatch theory can account for our results as follows. Suppose that the subject ignored the word hatchet (printed in white) on the prime trial, and then responded to the word hatchet (printed in blue) on the probe trial. According to the feature mismatch hypothesis, the word's identity (hatchet) would be associated with the color white on the prime trial. On the probe trial, however, it would be associated with the color blue. This mismatch would produce an inconsistency and a resultant delay in the processing of hatchet during the probe trial. In the identical condition, the ignored prime word always appeared in white and the target probe word always appeared in blue, so we would expect a mismatch to arise, yielding identity negative priming. There would be no semantic negative priming, though, because the mismatching color features between the probe target and the prime distractor would not reside in the same word in the related condition as they do in the identical condition.

Finally, Neill's episodic trace theory (Neill, Valdes, Terry, \& Gorfein, 1992) can also explain our results. According to this theory, it is not a mismatch between the features of the prime-trial distractor and the probe-trial target that produces negative priming; instead, interference derives from a competition between the correct response to the probe-display target and some sort of "nonresponse" previously associated with the ignored item in the prime display. That is, negative priming results when the probe-display target causes the subject to retrieve an item from memory that was recently associated with a different response requirement. This account can explain why negative priming was observed in the identical condition but not in the related condition of our experiment: Only the identical condition has the same item associated with two inconsistent responses. This would yield negative identity priming, but no semantic negative priming.

In conclusion, although neither the present results nor other extant results provide compelling support for any one of these theories over the other two (see the reviews by Fox, 1995; May et al., in press), the results of our study do converge nicely with those of earlier studies. It is clear that negative priming can span a variety of ordinarily powerful manipulations in the cognitive realm, a list that now includes quite different tasks on the prime and probe trials. Attention works not only because we can select some aspect of the environment or a recently processed episode, but also because we can exclude others. It is becoming increasingly clear that this exclusion of irrelevant information is a fundamental and pervasive feature of our ability to selectively attend.

\section{REFERENCES}

Battig, W. F., \& Montague, W. E. (1969). Category norms for verba items in 56 categories: A replication and extension of the Connecticut category norms. Journal of Experimental Psychology Monographs, 80, (3, Pt. 2), 1-45.

DALRYMPLE-AlFord, E. C., \& BUdAyr, B. (1966). Examination of some aspects of the Stroop color-word test. Perceptual \& Motor Skills, 23, 1211-1214.

Dark, V. J., Johnston, W. A., Myles-Worsley, M., \& Farah, M. J. (1985). Levels of selection and capacity limits. Journal of Experimental Psychology: General, 114, 472-497.

DrIVER, J., \& TIPPER, S. P. (1989). On the nonselectivity of "selective" seeing: Contrasts between interference and priming in selective attention. Journal of Experimental Psychology: Human Perception \& Performance, 15, 304-314.

Fox, E. (1994). Attentional bias in anxiety: A defective inhibition hypothesis. Cognition \& Emotion, 8, 165-195.

Fox, E. (1995). Negative priming from ignored distractors in visual selection: A review. Psychonomic Bulletin \& Review, 2, 145-173.

FuENTES, L. J., \& TUDELA, P. (1992). Semantic processing of foveally and parafoveally presented words in a lexical decision task. Quarterly Journal of Experimental Psychology, 45A, 299-322.

Graves, R., \& BRADLEY, R. (1987). Millisecond interval timer and auditory reaction time programs for the IBM PC. Behavior Research Methods, Instruments, \& Computers, 19, 30-35.

Graves, R., \& BRADLEY, R. (1988). More on millisecond timing and tachistoscope applications for the IBM PC. Behavior Research Methods, Instruments, \& Computers, 20, 408-412.

INHOFF, A. W. (1982). Parafoveal word perception: A further case against semantic processing. Journal of Experimental Psychology: Human Perception \& Performance, 8, 137-145.

INHOFF, A. W., \& RAYNER, K. (1980). Parafoveal word perception: A case against semantic preprocessing. Perception \& Psychophysics, 27, 457-464.

MalLeY, G. B., \& STRAYER, D. L. (1995). Effect of stimulus repetition on positive and negative identity priming. Perception \& Psychophysics, 57, 657-667. 
MAY, C. P., KANE, M. J., \& HASHER, L. (in press). Determinants of negative priming. Psychological Bulletin.

Milliken, B., TipPer, S. P., \& Weaver, B. (1994). Negative priming in a spatial localization task: Feature mismatching and distractor inhibition. Journal of Experimental Psychology: Human Perception \& Performance, 20, 624-646.

NEILL, W. T. (1977). Inhibitory and facilitatory processes in attention Journal of Experimental Psychology: Human Perception \& Performance, 3, 444-450.

NeILL, W. T., LISSNER, L. S., \& BeCK, J. L. (1990). Negative priming in same-different matching: Further evidence for a central locus of inhibition. Perception \& Psychophysics, 48, 398-400.

NeILL, W. T., \& VAldES, L. A. (1992). The persistence of negative priming: Steady-state or decay? Journal of Experimental Psychology: Learning, Memory, \& Cognition, 18, 565-576.

Neill, W. T., Valdes, L. A., Terry, K. M., \& Gorfein, D. S. (1992). The persistence of negative priming: II. Evidence for episodic trace retrieval. Journal of Experimental Psychology: Learning, Memory, \& Cognition, 18, 993-1000.

PARK, J., \& KanwISHER, N. (1994). Negative priming for spatial locations: Identity mismatching, not distractor inhibition. Journal of Experimental Psychology: Human Perception \& Performance, 20, 613 623.

TIPPER, S. P. (1985). The negative priming effect: Inhibitory priming by ignored objects. Quarterly Journal of Experimental Psychology, 37A, 571-590.

TIPPER, S. P. (1992). Selection for action: The role of inhibitory mechanisms. Current Directions in Psychological Science, 1, 105-109.

TIPPER, S. P., \& CRANSTON, M. (1985). Selective attention and priming: Inhibitory and facilitatory effects of ignored primes. Quarterly Journal of Experimental Psychology, 37A, 591-611.

TIPPER, S. P., \& DRIVER, J. (1988). Negative priming between pictures and words in a selective attention task: Evidence for semantic processing of ignored stimuli. Memory \& Cognition, 16, 64-70.

TIPPER, S. P., MACQUeEN, G. M., \& Brehaut, J. C. (1988). Negative priming between response modalities: Evidence for the central locus of inhibition in selective attention. Perception \& Psychophysics, 43, 45-52.

Tipper, S. P., Weaver, B., Cameron, S., Brehaut, J. C., \& Bastedo, J. (1991). Inhibitory mechanisms of attention in identification tasks:
Time-course and disruption. Journal of Experimental Psychology: Learning, Memory, \& Cognition, 17, 681-692.

TIPPER, S. P., WeaVer, B., \& Houghton, G. (in press). Behavioural goals determine inhibitory mechanisms of selective attention. Quarterly Journal of Experimental Psychology.

YEE, P. L. (1991). Semantic inhibition of ignored words during a figure classification task. Quarterly Journal of Experimental Psychology, 43A, 127-153.

\section{NOTE}

1. We actually tested two groups in the categorize-name condition, both containing 18 subjects. In the original group, the mean latencies and proportions of errors were, respectively, 752 and .054 for identical words, 750 and .085 for related words, and 732 and .069 for unrelated words. Although this resulted neither in a reliable interaction in the original $2 \times 2 \times 2$ ANOVA on negative priming difference scores (all $F \mathrm{~s}<1)$ nor in significant negative priming for the related condition alone $[t(17)=1.54, p=.14]$, we were troubled by the fact that the $r e$ lated negative priming effect $(18 \mathrm{msec})$ seemed of the same order as the identical one $(20 \mathrm{msec})$. Although the apparent negative priming in the related condition was largely due to 3 of the 18 subjects, we still felt that we should clear up this discrepancy.

The data reported in Table 1 and Figure 1 are from the second sample tested in the categorize-name condition and clearly show the same pattern as all of the other conditions, rather than the anomalous pattern of the first categorize-name sample tested. We also examined the data of all 36 subjects together in the categorize-name condition, and found that the overall $6 \mathrm{msec}$ of negative priming for the related condition was not reliable $[t(35)=0.67, p=.50]$. Further, almost exactly half $(19$, or $53 \%)$ of the 36 subjects showed negative priming for the related condition, as would be expected by chance, whereas most of them $(26$, or $72 \%$ ) showed negative priming in the identical condition. Thus, we are confident that the overall picture of negative priming only for the identical items in every task combination includes the categorizename condition.

(Manuscript received May 3, 1994; revision accepted for publication December $7,1994$. ) 